\title{
ECG Biometric Recognition: Permanence Analysis of QRS Signals for 24 Hours Continuous Authentication
}

\author{
Ruggero Donida Labati \#1, Roberto Sassi \#2, Fabio Scotti \#3 \\ \# Department of Computer Science, Università degli Studi di Milano \\ via Bramante, 65, I-26013 Crema (CR), Italy \\ ${ }^{1}$ ruggero.donida@unimi.it \\ 2 roberto.sassieunimi.it \\ 3 fabio.scottidunimi.it
}

\begin{abstract}
Recent studies regard the use of ECG signals for biometric recognition exploiting the possibility of these signals to be frequently recorded for long time periods without any explicit actions performed by the users during the acquisitions. This aspect makes ECG signals particularly suitable for continuous authentication applications. In this context, researches have proved that the QRS complex is the most stable component of the ECG signal.

In this paper, we perform a preliminary study on the persistency of QRS signals for continuous authentication systems. A recognition method based on multiple leads is proposed, and used to evaluate the persistency of the QRS complex in $\mathbf{2 4}$ hours Holter signals. This time interval can be considered as adequate for many possible applications in continuous authentication scenarios. The analysis is performed on a significantly large public Holter dataset and aims to search accurate matching and enrollment strategies for continuous authentication systems. At the best our knowledge, the results presented in this paper are based on the biggest set of ECG signals used to design continuous authentication applications in the literature.

Results suggest that the QRS complex is stable only for a relatively small time period, and the performance of the proposed recognition method starts decreasing after two hours.
\end{abstract}

\section{INTRODUCTION}

Recent studies in biometrics focus on the use of onedimensional physiological signals as biometric traits. Most of them are based on the electrocardiogram (ECG), electroencephalograms (EEG) [1], phonocardiogram (PCG) [2], and photoplethysmogram (PPG) [3,4].

With respect to other biometric traits, physiological signals present some important advantages [5].

- Physiological signals are more difficult to counterfeit with respect to samples of more commonly used biometric traits, like face, fingerprint, and voice. Physiological signals in fact, are more difficult to acquire using commonly diffused devices like cameras, microphones, and smartphones.

- Physiological signals can be acquired from all living individuals.

WIFS'2013, November 18-21, 2013, Guangzhou, China. ISBN 978-1-4673-5593-3 (c)2013 IEEE.
- The analysis of physiological signals can reveal other information related to the psychological states, and physiological and clinical status. The obtained information can be useful in some applicative contexts.

- Differently from most of the biometric traits, physiological signals can usually be acquired for long time periods without requiring that the users perform explicit actions, resulting particularly suitable for continuous authentication applications.

In this context, ECG is one of the most diffused and mature technologies in clinical analyses. Its use started in the early 1900s [6], and it became one of the first acquired signals in ambulatory monitoring since the 1970s.

Previous studies on ECG-based biometric systems show that ECG signals have sufficient discriminability for obtaining highly accurate results [7]. However, biometric systems have to overcome different non-idealities of the samples due to the heart rate variability, issues related to sensor placement, scalability, and the time-varying nature of the ECG signals [5].

Studies in the literature regarding the permanence of the ECG traits in long time periods report encouraging results [8]. However, a performance decrease can be usually observed by analyzing samples captured with sufficient time distance and uncontrolled conditions [5].

In these cases, researches have proved that the QRS complex of the ECG signal is most robust to these variations [9].

The QRS complex represents the depolarization of the right and left ventricles, and it corresponds to the biggest complex of the signal. It usually has a duration between 70 and $110 \mathrm{~ms}$ in a normal heartbeat. Other components of ECG signals are: the $\mathrm{P}$ wave, which describes the depolarization of the right and left atria, and the $\mathrm{T}$ wave, which reflects the ventricular repolarization. The peak points $\mathrm{P}, \mathrm{Q}, \mathrm{R}, \mathrm{S}, \mathrm{T}$ are usually called fiducial points. An example of these components of a signal describing an ideal heartbeat are shown in Fig. 1.

In this paper, we propose a preliminary study on continuous authentication systems based on ECG signals. The main goal of this work is to analyze the permanence of the QRS complex in order to evaluate the effect of possible continuous enrollment strategies in continuous authentication applications. 


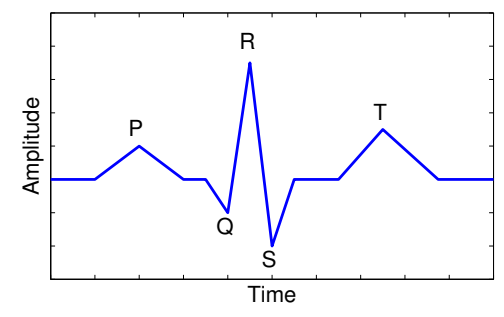

Fig. 1. Main components of an ideal heartbeat.

The design of a complete continuous authentication system is out of the scope of the paper.

The proposed biometric recognition method is therefore based on a simple correlation-based method in order to evaluate the effective similarity between the considered signals. The method exploits the information related to all the leads of the Holter by performing a fusion at the match score level [10] with the aim of obtaining accurate results.

The schema of the verification process is shown in Fig. 2. First, the QRS complex of every heartbeat is segmented by selecting a fixed-size time window centered in the $\mathrm{R}$ points. Then, a template describing multiple heartbeats is computed for each lead. The matching scores related to every lead are computed using a correlation-based strategy. Finally, a score fusion technique is applied to the previously computed similarity scores.

Experimental results show that the proposed method can obtain satisfactory accuracy using only the QRS complex of ECG signals. The persistency analysis of the QRS complex has then been performed on a dataset composed by 24 hours Holter signals recorded from 185 individuals, showing that the recognition performance is strictly related to the time passed between the acquisition of the compared signals.

The paper is structured as follows. Section II presents related works. In Section III, the proposed biometric recognition method based on the QRS complex is described. Section IV details the performed experiments and the obtained results. Finally, Section V summarizes the work.

\section{RELATED WORKS}

Most of the studies on ECG biometric recognition are based on a single lead [5] since the methods can be used in a greater variety of applicative contexts. Other studies use multiple leads $[11,12]$ because the greater quantity of information permits to increase the accuracy and reduce the noise effects. In this work, we have studied an approach based on multiple leads.

The recognition algorithms can be based on fiducial features or non-fiducial features [5] describing amplitude and frequency characteristics of the signals. The search of fiducial points in noisy long-time signals is particularly complex and can obtain poor results.

Many techniques in the literature extract information from all the components of ECG samples. Since the QRS complex is the most stable part of the ECG signal, methods that perform biometric recognition based only on this component have been studied. The technique presented in [13] is based on high-order Legendre Polynomials. The method described in [14] uses a matching strategy based on cross-correlation and Multilayer Perceptron (MLP) networks in order to evaluate samples captured in different applicative scenarios. In [15], non-fiducial features describing the signal frequency are extracted, Principal Component Analysis (PCA) is applied to reduce the feature set, and the matching is finally performed by using a two-levels classifier. The method presented in [16] performs the classification of QRS signals using multilayer perceptron and Radial Basis Function (RBF) neural networks.

At the best of our knowledge, in the literature there are only two studies on continuous authentication systems based on ECG signals [17,18]. The method presented in [17] performs authentications based on ECG signals every 35 heartbeats and the one detailed in [17] uses time intervals of $5 \mathrm{~s}$. These methods, however, have been evaluated on datasets composed by small sets of samples related to signals describing a maximum time period of 15 minutes.

There are also studies on the use of ECG signals in multimodal continuous authentication systems. In [19], ECG signals are combined with accelerometer-based data in order to increase the system accuracy.

Continuous authentication systems based on different biometric traits have also been studied [20]. Face characteristics are used in [21] to perform continuous recognition in applicative contexts in which the users are working in front to a computer. The system described in [22] is designed for a similar scenario, but it also uses soft biometric characteristics in order to obtain higher accuracy. A system based on fingerprint and face features is described in [23]. This system uses a holistic strategy to perform the fusion of the matching scores, which permits to fuse values obtained from samples acquired at different time instants. Another data fusion technique for continuous authentication systems is proposed in [24], and is based on fuzzy systems.

Most of these systems perform the enrollment once, but there are also studies that continuously update the stored templates [22].

In the literature, there are also examples of more complex strategies for periodically update the biometric database $[25,26]$.

\section{THE PROPOSED METHOD}

The proposed biometric recognition method only considers the QRS complex of ECG signals and uses multiple leads in order to increase the robustness to noise factors typically present in Holter acquisitions.

In the enrollment phase, continuous authentication methods can store the template once or periodically update the stored template in order to increase the recognition accuracy. This paper aims to evaluate the persistency of the QRS complex in order to analyze the advantages that could be offered by periodical enrollment strategies. Details on the performed tests are provided in Section IV. 


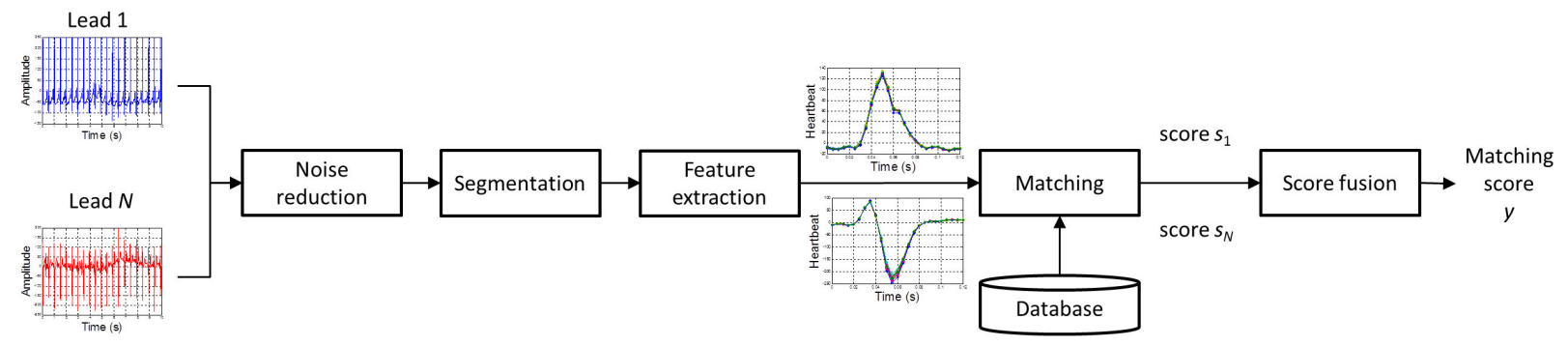

Fig. 2. Schema of the proposed recognition method.

In the verification and identification phases, the method computes distinctly the matching scores related to every lead. The obtained information is finally fused to compute the recognition result. The used feature extraction and matching algorithms are similar to the ones that we have designed for PPG signals [27]. They are based on a simple correlation technique since this work does not aim to obtain extremely accurate results, but its principal goal is to analyze the persistency of the QRS complex for designing continuous authentication techniques.

The verification algorithm applied to every lead can be divided into the sequent steps:

1) noise reduction;

2) segmentation;

3) feature extraction;

4) matching.

The obtained matching scores are finally fused by using wellknown strategies in the literature.

\section{A. Noise reduction}

First, the noise introduced by the $50 \mathrm{~Hz}$ electrical component is removed using a notch IIR filter, applied also after the time reversal to avoid phase distortions. Finally, the signal's baseline is normalized by applying a third order high-pass Butterworth filter with cutoff frequency of $0.5 \mathrm{~Hz}$ [28].

\section{B. Segmentation}

The QRS signal segmentation is then performed in order to obtain a matrix $H$ in which the pulses pertaining to every heartbeat of a sample $S$ are stored in distinct rows. For each heartbeat, the signal representing the QRS complex is obtained by selecting a fixed time interval centered in the $\mathrm{R}$ point. For each $\mathrm{R}$ point $i$, the QRS signal is computed as:

$$
\mathrm{QRS}\left(1 \ldots t_{1}+t_{2}\right)=S\left(i-t_{1} \ldots i+t_{2}\right),
$$

where $t_{1}$ and $t_{2}$ are empirically estimated values.

We employed the list of $\mathrm{R}$ fiducial points supplied with the database, which have been obtained from an automatic labeling software (Vision Premier, SpaceLab-Burdick Inc.) and manually reviewed and adjudicated. In order to avoid improperly located beats and artifacts, QRS complexes with a cross-correlation coefficient smaller than 0.9 with a common average pattern are excluded. This is a strategy commonly employed in biomedical studies [29].

\section{Feature extraction}

The used biometric templates represent the $m$ QRS signals with maximum cross-correlation values between every QRS and the average QRS pattern. In the cases in which a number of beats of sufficient quality is not found, the templates are composed by a smaller number of heartbeats.

In our tests, the computation of templates composed by sets of signals obtained better accuracy with respect to the more commonly adopted strategy that performs the computation of a single signal representing the mean heartbeat. This method, however, is less efficient in terms of computational time.

In practice, starting from a matrix $H$ composed by $n$ QRS signals, the template $T$ is computed by using an iterative algorithm. For each iteration, the signal $\overline{\mathrm{QRS}}$ representing the amplitude of the average QRS pattern is computed as:

$$
\overline{\mathrm{QRS}}(j)=\sum_{i=1}^{n} H(i, j) / n .
$$

The cross-correlation between $\overline{\mathrm{QRS}}$ and every signal of $H$ is then computed, obtaining the vector $C$. The $m$ QRS signals corresponding to the maximum values of $C$ are stored in the template $T$. Signals with correlation values less than an empirically estimated threshold $t_{c}$ are removed from $T$ since they present noise or excessive variations from the shape of the mean signal $\overline{\mathrm{QRS}}$.

Two iterations are performed in order to reduce noise effects. The goal of the second iteration is to delete possible noisy heartbeats, and it considers $H=T$. The number $m$ is equal for each iteration.

\section{Matching}

This step computes a similarity score $s$ between two templates $T_{A}$ and $T_{B}$ related to the same lead.

The maximum cross-correlation between every QRS signal pertaining to $T_{A}$ and every signal of $T_{B}$ is computed, obtaining the similarity matrix $M$. As an example, Fig. 3 shows a matrix $M$ computed during a genuine identity comparison of templates composed by two QRS signals.

Different score fusion strategies have been considered for the computation of the score $s$.

- Fusion $_{A}: s=\operatorname{mean}(M)$.

- Fusion $_{B}: s=\operatorname{median}(M)$.

- Fusion $_{C}: s=75$ th percentile $(M)$. 
(a)
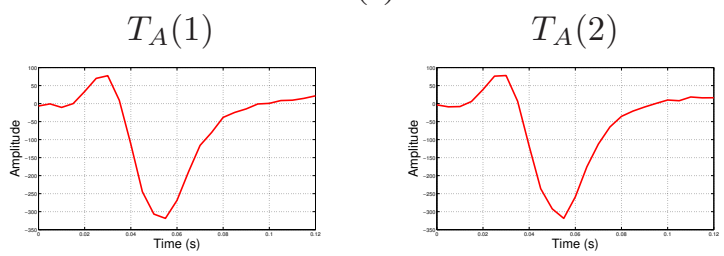

(b)
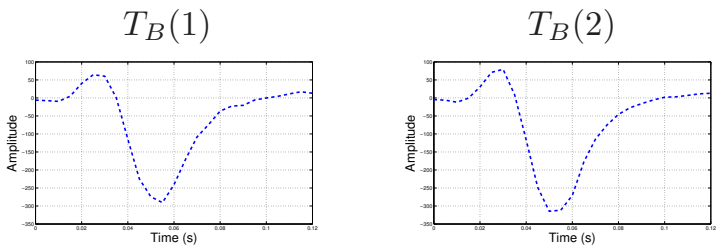

(c)

\begin{tabular}{l|l|l|} 
& \multicolumn{1}{c}{$T_{B}(1)$} & $T_{B}(2)$ \\
\cline { 2 - 3 }$T_{A}(1)$ & 0.9955 & 0.9950 \\
\cline { 2 - 3 }$T_{A}(2)$ & 0.9965 & 0.9961 \\
\hline
\end{tabular}

Fig. 3. Example of similarity matrix $M$ obtained from a genuine identity comparison between the templates $T_{A}$ and $T_{B}$, which are composed by two signals $(n=2)$ : (a) template $T_{A}$; (a) template $T_{B}$; similarity matrix $M$.

- Fusion $_{D}: s=90$ th percentile $(M)$.

- Fusion ${ }_{E}: s=95$ th percentile $(M)$.

- Fusion $_{F}: s=\max (M)$.

\section{E. Score Fusion}

Considering $N$ similarity scores $s_{i}$ obtained from signals acquired by $N$ leads at the same time instant, the final similarity score values $y$ can be obtained by using different score fusion strategies [10]. In this work, we have experimentally evaluated the approaches described in Subsection III-D.

\section{EXPERIMENTAL RESULTS}

The performed experiments aim to analyze the permanence and stability of the biometric features extracted from the QRS complex in ECG signals on a time period of a day. The goal of these tests is to provide preliminary results necessary to design effective matching and enrollment strategies for continuous authentication systems.

In order to properly design the studied algorithms, we have evaluated the verification accuracy of the proposed biometric recognition method in different configurations, encompassing the number of heartbeats used to compute the templates, six data fusion strategies for the matching step, and six strategies for the score fusion step. Then, the permanence evaluation has been performed by analyzing the matching scores obtained by the biometric technique on samples acquired at different time intervals. This test only aims to evaluate the permanence of the QRS complex, and it is not based on specifically designed methods for periodic enrollment, which should be studied in future works.

The dataset employed has been extracted from the E-HOL03-0202-003 (Intercity Digital Electrocardiogram Alliance IDEAL) database [30]. The experimental dataset is composed by 185 digital 24-hours Holter recordings (SpaceLab-Burdick Inc.) from 185 individuals. The series are actually 202, but for 17 of them one of the leads is corrupted by artifacts and noise, and they have been excluded from our analysis. The acquisitions have been performed without any restrain or control on the activities performed. Males and females are equally distributed. The Electrocardiograms have been acquired using a three pseudo-orthogonal leads configuration (X, Y and $\mathrm{Z}$ ). The signals have sampling frequency equal to $200 \mathrm{~Hz}$ and amplitude resolution equal to $10 \mu \mathrm{V}$.

The parameters used by the proposed method have been empirically set on the used dataset, and are $t_{1}=50 \mathrm{~ms}, t_{2}=$ $70 \mathrm{~ms}$, and $t_{c}=0.9$.

\section{A. Accuracy evaluation}

In order to evaluate the accuracy of the proposed biometric recognition method in verification scenarios, we have created a dataset representing samples captured with time distance of about 3 hours $\left(\mathrm{DB}_{A}\right)$. The dataset $\mathrm{DB}_{A}$ is composed by 1307 samples. For each individual, the number of samples varies from 7 to 8 .

We have then evaluated the performance of the proposed recognition method in different configurations on $\mathrm{DB}_{A}$, performing 8070 genuine identity comparisons and 1696260 impostor identity comparisons. The tested parameters of the proposed method are the number $m$ of QRS signals composing the templates (with values $2,4,8,16$ ) and the information fusion strategies. The best results have been obtained by using $m=16$ and the strategy Fusion $F$ for both the feature extraction and data fusion steps. The obtained Detection Error Tradeoff (DET) curve is shown in Fig. 4, and the False Match Rate (FM) and False Non Match Rate (FNM) in different working points of the curve are reported in Table I.

The obtained results show that the method can obtain sufficient accuracy to be used in different applicative scenarios, reporting an Equal Error Rate (EER) of 5.36\%.

\section{B. Permanence analysis}

In order to analyze the permanence of QRS signals in 24 hours Holter acquisitions, we have evaluated the accuracy of the proposed biometric recognition method in an ideal continuous authentication scenario in which verifications are performed at fixed time intervals and the enrolled templates are updated after every verification. Differently from traditional procedures used for the accuracy evaluation of biometric recognition algorithms, which do not consider the time passed between biometric acquisitions [31], this test permits to analyze the stability and persistency of the used features during a defined time interval. The analysis has been performed considering both applications that compute verifications at short and long time intervals.

The tests have been performed on the dataset $\mathrm{DB}_{B}$, obtained by dividing all the available Holter acquisitions in samples with a duration of 5 minutes. This dataset is composed by 50045 samples. For each individual, the number of samples varies from 260 to 290 . 


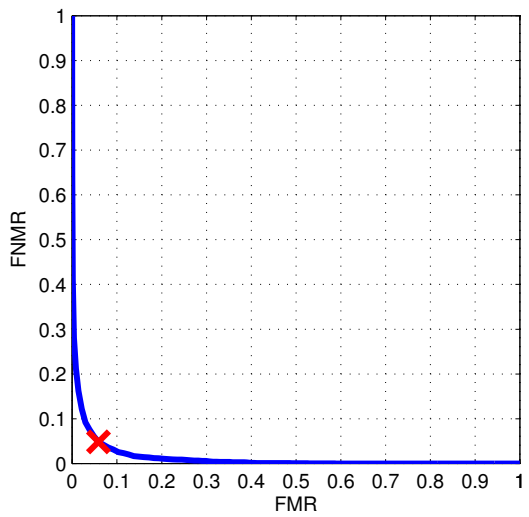

Fig. 4. DET curve of the proposed method on $\mathrm{DB}_{A}$. The red cross marks the position of the EER.

TABLE I

BEST RESULTS OF THE REALIZED METHOD DB $A$.

\begin{tabular}{llc}
\hline \hline DET point & $\mathrm{DB}_{A}$ \\
\hline EER (\%) & & 5.36 \\
FMR (\%) & FNMR $=5 \%$ & 6.11 \\
FMR (\%) & FNMR $=10 \%$ & 3.15 \\
FMR (\%) & FNMR $=15 \%$ & 1.52 \\
FNMR (\%) & FMR $=5 \%$ & 5.87 \\
FNMR (\%) & FMR $=10 \%$ & 2.94 \\
FNMR (\%) & FMR $=15 \%$ & 2.12 \\
\hline \hline
\end{tabular}

In the performed tests, we have computed genuine identity comparisons for each individual $i$. For each time instant $j$, we have computed the matching score between the template $T_{(i, j)}$ and the template $T_{\left(i, j-\Delta_{t}\right)}$ obtained at the time instant $j-\Delta_{t}$ :

$$
G_{(i, j)}=\operatorname{matching}\left(T_{(i, j)}, T_{\left(i, j-\Delta_{t}\right)}\right) .
$$

In order to reduce the computational time needed to perform the tests, the reported results have been computed using the impostor matching scores obtained from $\mathrm{DB}_{A}$. For this preliminary analysis, we have assumed that the impostor matching scores obtained with a resampling step of three hours from the set of Holter acquisitions (1696260 over 2493586236 possible impostor comparisons) can sufficiently approximate the shape of the impostor distribution.

1) Short time interverval: We have analyzed the accuracy of the system varying the value of $\Delta_{t}$ from 5 minutes (consecutive samples) to 95 minutes, with a step of 15 minutes. Fig. 5 and Table II report the obtained results.

Fig. 6 and Table III show that QRS signals can be considered permanent in a short time period of 90 minutes. The mean EER is $1.59 \%$, and the standard deviation of the EER values is $0.52 \%$.

2) Long time interverval: we have analyzed the accuracy of the system varying the value of $\Delta_{t}$ from 5 minutes (consecutive samples) to 10 hours and 5 minutes, with a step of two hours. The obtained results are reported in Fig. 6 and Table III.

From the obtained results, it is possible to observe that the obtained EER values tend to increase with the increasing

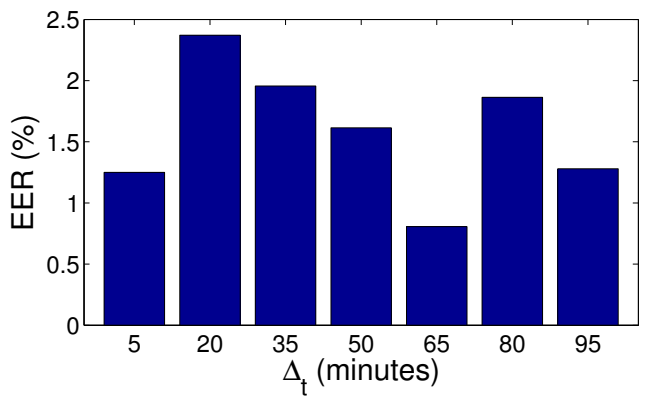

Fig. 5. EER obtained at different time distances $\Delta_{t}$ on $\mathrm{DB}_{B}$ in a short time period. The obtained accuracy measured as ERR is bounded in an interval between $0.81 \%$ and $1.96 \%$, with mean equal to $1.59 \%$, and the standard deviation equal to $0.52 \%$.

TABLE II

EER OBTAINED AT DIFFERENT $\Delta_{t}$ IN A SHORT TIME PERIOD.

\begin{tabular}{ccc}
\hline \hline$\Delta_{t}$ & Genuine comparisons & EER (\%) \\
\hline 5 & 50505 & 1.25 \\
20 & 51245 & 2.37 \\
35 & 51615 & 1.96 \\
50 & 50135 & 1.61 \\
65 & 47545 & 0.81 \\
80 & 46065 & 1.86 \\
95 & 47915 & 1.28 \\
\hline \hline
\end{tabular}

of $\Delta_{t}$. The accuracy decreasing, anyway, is not linear since other aspects can influence the obtained results: quality of the samples; activities performed by the users; emotional states.

These results suggest that QRS signals are not completely permanent during 24 hours.

In order to increase the accuracy of continuous authentication systems, more robust features and automatic techniques for continuous enrollment $[25,26]$ should be adopted.

\section{CONCLUSION}

This paper presented a permanence analysis of the QRS complex in 24 hours Holter acquisitions and investigated different matching and enrollment strategies for continuous authentication systems.

The permanence analysis have been performed using a method based on multiple leads and correlation-based algorithms.

The obtained results showed a verification error measured as Equal Error Rate (ERR) of $5.36 \%$ on a dataset of samples acquired in 24 hours. The persistency analysis in short time periods exhibited that samples acquired with time differences of less than 95 minutes obtained sufficient stability, with mean EER equal to $1.59 \%$. Differently, the persistency analysis performed on long time periods obtained a gradual accuracy decreasing, with worst EER equal to $8.55 \%$.

In order to design accurate continuous authentication systems, more robust features and periodical enrollment strategies should be adopted. In future work, we will exploit the obtained information in order to design a complete continuous authentication system based on ECG signals. 


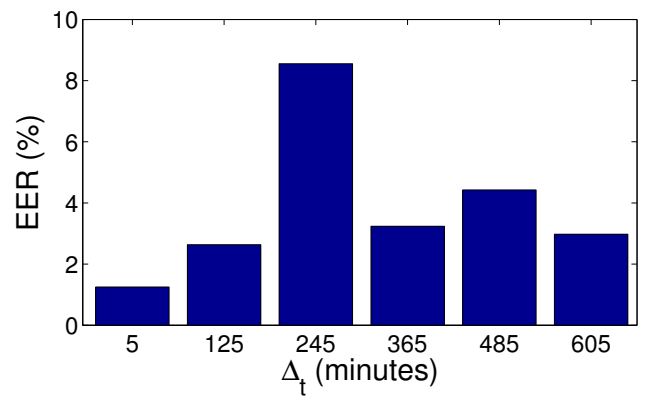

Fig. 6. EER obtained at different time distances $\Delta_{t}$ on $\mathrm{DB}_{B}$ in a long time period. It is possible to observe that the accuracy decreases at the increasing of $\Delta_{t}$.

TABLE III

EER OBTAINED AT DIFFERENT $\Delta_{t}$ IN A LONG TIME PERIOD.

\begin{tabular}{ccc}
\hline \hline$\Delta_{t}$ & Genuine comparisons & EER (\%) \\
\hline 5 & 50505 & 1.25 \\
125 & 46990 & 2.64 \\
245 & 44215 & 8.55 \\
365 & 35335 & 3.24 \\
485 & 33485 & 4.43 \\
605 & 30895 & 2.98 \\
\hline \hline
\end{tabular}

\section{ACKNOWLEDGEMENTS}

This work was supported in part by the Italian Ministry of Research within PRIN 2010-2011 project "GenData 2020" (2010RTFWBH).

Data used for this research were provided by the Telemetric and Holter ECG Warehouse of the University of Rochester (THEW), NY.

\section{REFERENCES}

[1] W. Khalifa, A. Salem, M. Roushdy, and K. Revett, "A survey of EEG based user authentication schemes," in Proc. of the 8th Int. Conf. on Informatics and Systems (INFOS), 2012, pp. 55 - 60.

[2] F. Beritelli and S. Serrano, "Biometric identification based on frequency analysis of cardiac sounds," IEEE Transactions on Information Forensics and Security, vol. 2, no. 3, pp. 596 - 604, 2007.

[3] Y. Y. Gu, Y. Zhang, and Y. Zhang, "A novel biometric approach in human verification by photoplethysmographic signals," in Proc. of the 4th IEEE Annual Int. Conf. of the Engineering in Medicine and Biology Society (EMBS), 2003, pp. $13-14$

[4] Y. Y. Gu and Y. Zhang, "Photoplethysmographic authentication through fuzzy logic," in Proc. of the IEEE EMBS Asian-Pacific Conf. on Biomedical Engineering, 2003, pp. 136 - 137.

[5] I. Odinaka, P.-H. Lai, A. Kaplan, J. O'Sullivan, E. Sirevaag, and J. Rohrbaugh, "ECG biometric recognition: A comparative analysis," IEEE Transactions on Information Forensics and Security, vol. 7, no. 6, pp. $1812-1824,2012$.

[6] L. Sörnmo and P. Laguna, Bioelectrical Signal Processing in Cardiac and Neurological Applications. Elsevier Academic Press, June 2005.

[7] J. Irvine and S. Israel, "A sequential procedure for individual identity verification using ECG," EURASIP Journal on Advances in Signal Processing, no. 1, 2009.

[8] I. Odinaka, P.-H. Lai, A. Kaplan, J. O'Sullivan, E. Sirevaag, S. Kristjansson, A. Sheffield, and J. W. Rohrbaugh, "ECG biometrics: A robust short-time frequency analysis," in Proc. of the 2010 IEEE International Workshop on Information Forensics and Security (WIFS), 2010, pp. 1 6.

[9] S. A. Israel, J. M. Irvine, A. Cheng, M. D. Wiederhold, and B. K. Wiederhold, "ECG to identify individuals," Pattern Recognition, vol. 38, no. 1 , pp. $133-142,2005$.
[10] A. Ross and A. K. Jain, "Multimodal Biometrics: an overview," 2004, pp. $1221-1224$

[11] C. Ye, M. Coimbra, and B. Kumar, "Investigation of human identification using two-lead electrocardiogram (ECG) signals," in Proc. of the 2010 Fourth IEEE Int. Conf. on Biometrics: Theory Applications and Systems (BTAS), 2010, pp. $1-8$.

[12] F. Poree, J. Y. Bansard, G. Kervio, and G. Carrault, "Stability analysis of the 12-lead ECG morphology in different physiological conditions of interest for biometric applications," in Computers in Cardiology, 2009, pp. $285-288$.

[13] I. Khalil and F. Sufi, "Legendre polynomials based biometric authentication using QRS complex of ECG," in Proc. of the 2008 Int. Conf. on Intelligent Sensors, Sensor Networks and Information Processing (ISSNIP), 2008, pp. 297 - 302.

[14] K. Sidek, I. Khalil, and M. Smolen, "ECG biometric recognition in different physiological conditions using robust normalized QRS complexes," in Computing in Cardiology (CinC), 2012, pp. 97 - 100.

[15] L. S. Hou, K. Subari, and S. Syahril, "QRS-complex of ECG-based biometrics in a two-level classifier," in Proc. of the 2011 IEEE Region 10 Conf-(TENCON), 2011, pp. 1159 - 1163.

[16] V. Mai, I. Khalil, and C. Meli, "ECG biometric using multilayer perceptron and radial basis function neural networks," in Proc. of the 2011 Annual Int. Conf. of the IEEE Engineering in Medicine and Biology Society $(E M B C), 2011$, pp. $2745-2748$.

[17] M. Guennoun, N. Abbad, J. Talom, M. Rahman, and K. El-Khatib, "Continuous authentication by electrocardiogram data," in Proc. of the IEEE Toronto Int. Conf. on Science and Technology for Humanity (TICSTH), 2009, pp. 40-42.

[18] R. Matta, J. Lau, F. Agrafioti, and D. Hatzinakos, "Real-time continuous identification system using ECG signals," in Proc. of the 24th Canadian Conference on Electrical and Computer Engineering (CCECE), 2011, pp. $1313-1316$.

[19] J. C. Sriram, M. Shin, T. Choudhury, and D. Kotz, "Activity-aware ECGbased patient authentication for remote health monitoring," in Proc. of the 2009 int. conf. on Multimodal interfaces, 2009, pp. 297 - 304.

[20] I. Traore and A. Ahmed, Continuous Authentication Using Biometrics: Data, Models, and Metrics. IGI Global, 2011.

[21] K. Niinuma and A. K. Jain, "Continuous user authentication using temporal information," vol. 7667, 2010.

[22] K. Niinuma, U. Park, and A. Jain, "Soft biometric traits for continuous user authentication," IEEE Transactions on Information Forensics and Security, vol. 5, no. 4, pp. 771 - 780, 2010.

[23] T. Sim, S. Zhang, R. Janakiraman, and S. Kumar, "Continuous verification using multimodal biometrics," IEEE Transactions on Pattern Analysis and Machine Intelligence, vol. 29, no. 4, pp. 687 - 700, 2007.

[24] A. Azzini, S. Marrara, R. Sassi, and F. Scotti, "A fuzzy approach to multimodal biometric continuous authentication," Fuzzy Optimization and Decision Making, vol. 7, no. 3, pp. 243 - 256, September 2008.

[25] A. Rattani, B. Freni, G. Marcialis, and F. Roli, "Template update methods in adaptive biometric systems: A critical review," in Advances in Biometrics, ser. Lecture Notes in Computer Science, M. Tistarelli and M. Nixon, Eds. Springer Berlin Heidelberg, 2009, vol. 5558, pp. 847 -856 .

[26] A. Rattani, G. L. Marcialis, and F. Roli, "Biometric system adaptation by self-update and graph-based techniques," Journal of Visual Languages and Computing, 2012.

[27] A. Bonissi, R. Donida Labati, L. Perico, R. Sassi, F. Scotti, and L. Sparagino, "A preliminary study on continuous authentication methods for photoplethysmographic biometrics," in Proc. of the 2013 IEEE Workshop on Biometric Measurements and Systems for Security and Medical Applications (BioMS 2013), SUBMITTED.

[28] J. Proakis and D. Manolakis, Digital signal processing. Pearson Prentice Hall, 2007.

[29] R. Sassi and L. Mainardi, "An estimate of the dispersion of repolarization times based on a biophysical model of the ECG," IEEE Transactions on Biomedical Engineering, vol. 58, no. 12, pp. 3396 - 3405, 2011.

[30] University of Rocher Medical Center, Telemetric and Holter ECG Warehouse, "E-hol-03-0202-003," http://thew-project.org/Database/E-HOL03-0202-003.html.

[31] M. Gamassi, M. Lazzaroni, M. Misino, V. Piuri, D. Sana, and F. Scotti, "Quality assessment of biometric systems: a comprehensive perspective based on accuracy and performance measurement," IEEE Transactions on Instrumentation and Measurement, vol. 54, no. 4, pp. 1489 - 1496, August 2005. 